\title{
ABANDONO DA TERAPIA DO TRATAMENTO DA TUBERCULOSE MULTIRRESISTENTE: DESAFIOS PARA OS PROFISSIONAIS DA SAÚDE
}

\author{
ABANDONMENT OF MULTIRRESISTANT \\ TUBERCULOSIS TREATMENT THERAPY: \\ CHALLENGES FOR HEALTH PROFESSIONALS
}

Debora Paula Ferreira Volpe Maria Catarina Salvador Mota?

\begin{abstract}
RESUMO
Objetivo: Descrever as características dos casos de abandono do tratamento de tuberculose em pacientes que desenvolveram TBMR. Método: Estudo de abordagem quantitativa, desenvolvido em um hospital terciário de referência para tratamento de TBMR localizada no municipio do Rio de Janeiro. Foi utilizado instrumento estruturado com informaçóes de oitenta prontuários entre os meses de fevereiro a dezembro de 2017. A análise dos dados foi realizada através do software SPSS. Resultados: Foi possível verificar que dos 80 pacientes estudados: 35\% abandonaram tratamento anterior de Tuberculose, a média de idade foi de 43,6 anos; 67,5\% dos pacientes eram do sexo masculino e $42,5 \%$ pardos. Conclusão:As evidências desse estudo apontam que é necessária a identificação precoce de pacientes que apresentam maior risco para abandono do tratamento, bem como a criação de um modelo de assistência voltado ao perfil dos usuários que abandonam o tratamento, com uma prática participativa dos profissionais de saúde, com grupos educativos que promovam açóes preventivas, identificando, estudando estratégias para a superação de barreiras ligadas à realidade, de vida individual e coletiva. Palavras-chave: Tuberculose Resistente a Múltiplos Medicamentos. Adesão à medicação. Terapêutica.
\end{abstract}

\begin{abstract}
Objective: To describe the characteristics about the cases of abandonment on tuberculosis treatment in patients who developed multirresistant tuberculosis (TBMR). Method: A quantitative approach, developed in a tertiary reference hospital for the treatment of TBMR located in Rio de Janeiro. A structured instrument with information from eighty medical records was used between February and December 2017. Data analysis was performed using SPSS software. Results: It was possible to verify that about the 80 patients studied, $35 \%$ abandoned previous treatment of Tuberculosis, the mean age was 43.6 years, $67.5 \%$ of the patients were male, 42.5\% were mixed race. Conclusion: Evidence from this study indicates that it is necessary to identify patients at major risk for treatment abandonment, as well as to create a model of assistance aimed at the profile of users who abandon treatment, with a participative practice of the professionals of health, with educational groups that promote preventive actions, identifying, studying strategies for overcoming barriers linked to reality, individual and collective life.
\end{abstract}

Keywords: Tuberculosis, Multidrug-Resistant. Medication adherence. Therapeutics.

Enfermeira. Mestre em Ciências Biológicas. Escola de Enfermagem Anna Nery - Universidade Federal do Rio de Janeiro. E-mail: pfv.debora@ gmail.com

Enfermeira. Doutora em Enfermagem. Escola de Enfermagem Anna Nery - Universidade Federal do Rio de Janeiro. 


\section{INTRODUÇÃO}

Uma das principais preocupaçóes com respeito à tuberculose é a redução das taxas de abandono de tratamento. No Brasil, a taxa de abandono é alta, situa-se em torno de $17 \%$, porém, em muitas regiôes, atingem níveis mais elevados. Isso leva ao não rompimento da cadeia de transmissão, pois as pessoas com Tuberculose (TB) que não aderem à terapêutica permanecem doentes e continuam como fonte de contágio. Além disso, o abandono do tratamento leva à resistência medicamentosa e à recidiva da doença, as quais impóem dificuldades ao processo de cura do paciente, aumentando o tempo e o custo do tratamento ${ }^{(1-3)}$.

O abandono do próprio tratamento é descrito pelo Ministério da Saúde como a ausência do usuário por mais de 30 dias consecutivos após a data aprazada para retorno, em nível nacional, entre as Unidades da Federação do país, a proporção de abandono variou de 2,8 a 15,9\% no ano de $2014^{(4)}$. Essa ruptura na adesão acaba por gerar acentuado impacto nos indicadores de incidência, resistência a múltiplas drogas, doenças, agravos associados, e aumento das taxas de mortalidade ${ }^{(5)}$. As causas conhecidamente associadas à interrupção do tratamento envolvem fatores intrínsecos ao usuário como exemplo o uso de álcool e outras drogas, falsa impressão de cura e desconforto causado pela falta de alimento e fatores extrínsecos como modalidade de tratamento aplicado e operacionalização dos serviços de saúde ${ }^{(6)}$.

Ainda como ingrediente potente para o desafio do controle da tuberculose, surge a multidroga resistência para o tratamento. A Organização Mundial de Saúde estima que todos os anos em torno de 480,000 pessoas desenvolvem tuberculose TBMR. Em 2013, apenas 20\% dessas pessoas receberam regimes de tratamento recomendados $^{(7)}$. Daqueles doentes que são tratados apenas $10 \%$ conseguem a cura, podendo ainda apresentar formas mais resistentes (TBXDR) e com possibilidade de cura improvável e consequente morte ${ }^{(8)}$.
No entanto, o crescimento do número desses casos vem provocando preocupação em todo o mundo, e essa forma clínica de multirresistência vem sendo cada vez mais relacionada com a deficiência dos sistemas de saúde e apresenta-se nos dias de hoje como um grande desafio global do controle da $\mathrm{TB}^{(9)}$. $\mathrm{O}$ advento dos antibióticos para o tratamento da tuberculose (TB) corresponde a um elevado avanço na luta contra a doença. Porém, desde a sua primeira utilização, a antibioticoterapia tem sido associada com o aparecimento de linhagens de Micobacterium tuberculosis resistentes as drogas ${ }^{(10)}$.

A TB-XDR é uma ameaça de extrema importância para a saúde pública. A sua própria existência é o reflexo da ausência de controle da TB e da falta de implementação das medidas recomendadas no WHO's Stop TB Strategy, que deveriam minimizar a emergência de resistência aos fármacos ${ }^{(8)}$.

O número de casos de tuberculose multirresistente (TBMR) e de tuberculose extensivamente resistente aos medicamentos (TB-XDR) aumentou significativamente no mundo(11). Dados recentes mostram que o número de casos de TBMR triplicou entre 2009 e 2013, em 2013, os casos de TBMR representaram $3,5 \%$ de todos os novos casos de tuberculose e $20,5 \%$ de todos os casos de tuberculose previamente tratados, já os casos de TB-XDR representaram $9 \%$ de todos os casos de TB-MR notificados em 100 países $^{(12)}$.

Diante do exposto, o objetivo deste estudo foi analisar a ocorrência do abandono de tratamento da tuberculose e a posterior complicaçáo para o desenvolvimento de Tuberculose Multirresistente em uma instituição terciária de referência para tratamento de Tuberculose Multirresistente, localizado no município do Rio de Janeiro.

\section{MÉTODO}

Estudo descritivo, transversal de abordagem quantitativa. Desenvolvido a partir de informações obtidas em 80 prontuários de pacientes que 
foram encaminhados com TBMR, no Serviço de Tisiologia do Hospital Universitário Clementino Fraga Filho (HUCFF). O HUCFF é um Hospital de referência para tratamento de tuberculose multirresistente, localizado no campus da Universidade Federal do Rio de Janeiro (UFRJ), que atualmente recebe pacientes diagnosticados com tuberculose multirresistente referente as áreas programáticas 2.2. No momento da coleta, 80 pacientes tinham dado entrada no HUCFF para tratamento de TBMR, sendo então utilizado todos os prontuários, para a coleta de dados, uma vez que todos atendiam o critério de inclusão do estudo.

O estudo foi realizado durante o período de fevereiro a dezembro de 2017 e os dados foram obtidos mediante análise dos prontuários.

A populaçáo foi composta por pacientes pertencente a Área Programática (AP) 2.2 que são encaminhados ao HUCFF para tratamento de TBMR. A (AP) 2.2 tem o hospital de estudo como referência para o tratamento da TBMR e abrange 7 bairros: Praça da Bandeira, Tijuca, Alto da Boa Vista, Maracanã, Vila Isabel, Andaraí e Grajaú.

A amostra foi delimitada conforme os seguintes critérios de inclusão: pacientes com diagnóstico de TBMR, idade igual ou superior a 18 anos, e em tratamento com esquema básico preconizado pelo Programa Nacional de Combate à Tuberculose (PNCT). Como critérios de exclusão, não foram elegíveis os prontuários de usuários que apresentaram incompletude de informaçôes e dificuldade de compreensão.

Para a coleta de dados, foi utilizado um questionário estruturado para a avaliação socio demográfica no qual as variáveis investigadas foram: sexo, cor da pele, idade, escolaridade, forma de $\mathrm{TB}$, resultado de exame anti-HIV, coinfecção HIV-Aids, e presença de outras comorbidades.
Também foram pesquisados dados sobre a forma de entrada no programa, se eram pacientes virgens de tratamento (caso novo) ou se eram pacientes com tratamento prévio para tuberculose (recidivas). Além da pesquisa de dados como a forma clínica predominante da tuberculose e o acompanhamento com a estratégia DOTS.

Os dados obtidos foram codificados e digitados em planilha eletrônica Microsoft Excel 2010 e analisados com apoio do pacote estatístico para as ciências sociais SPSS versão 22.0. Foram tratados com estatística descritiva e apresentados em tabelas de frequência, com valores absolutos (n) e percentuais (\%) para as variáveis categóricas.

Todos os cuidados éticos que regem pesquisas com seres humanos foram observados e respeitados, segundo a Resolução 466/2012, e na Normativa 001/2013 regulamentada pelo Conselho Nacional de Saúde.

O estudo foi aprovado pelo Comitê de Ética em Pesquisa da Escola de Enfermagem Anna Nery (EEAN), da Universidade Federal do Rio de Janeiro (UFRJ), em 31 de maio de 2016, tendo como número de Protocolo CAAE 54091116.7.0000.5238.

\section{RESULTADOS}

A Tabela 1 apresenta que entre os 80 pacientes investigados, $67,5 \%$ eram do sexo masculino e $32,5 \%$ do sexo feminino, a maioria de cor parda 42,5\%. Quando analisado o nível de escolaridade, percebeu-se que do total de pacientes estudados, $40 \%$ tinham entre quatro e sete anos de estudo e $42,5 \%$ entre oito e onze anos de estudo, este tempo também sendo observado entre os que abandonaram o tratamento.

Quadro 1. Perfil socio demográfico e clínico dos pacientes com TBMR

\begin{tabular}{cccccc}
\hline Variáveis & & \multicolumn{2}{c}{ Casos Notificados } & \multicolumn{2}{c}{ Casos de Abandono } \\
\hline \multirow{2}{*}{ Sexo } & & $\mathrm{N}$ & $\%$ & $\mathrm{~N}$ & $\%$ \\
& Masculino & 54 & 67,5 & 20 & 71,4 \\
& Feminino & 26 & 32,5 & 8 & 28,6 \\
\hline
\end{tabular}


Continuaçáo - Quadro 1 - Perfil socio demográfico e clínico dos pacientes com TBMR

\begin{tabular}{|c|c|c|c|c|c|}
\hline \multirow{2}{*}{$\frac{\text { Variáveis }}{\text { Cor }}$} & \multirow[b]{2}{*}{ Branco } & \multicolumn{2}{|c|}{ Casos Notificados } & \multicolumn{2}{|c|}{ Casos de Abandono } \\
\hline & & 24 & 30 & 10 & 35,7 \\
\hline & Pardo & 34 & 42,5 & 8 & 28,6 \\
\hline & Negro & 22 & 27,5 & 10 & 35,7 \\
\hline \multirow[t]{4}{*}{ Idade } & $0-20$ anos & 6 & 7,5 & 2 & 7,2 \\
\hline & $21-40$ anos & 42 & 52,5 & 12 & 42,9 \\
\hline & $41-60$ anos & 16 & 20 & 8 & 28,5 \\
\hline & 61 ou mais & 16 & 20 & 6 & 21,4 \\
\hline \multirow[t]{4}{*}{ Escolaridade } & $0-3$ anos & 8 & 10 & 2 & 7,2 \\
\hline & 4-7 anos & 32 & 40 & 20 & 71,4 \\
\hline & 8-11 anos & 34 & 42,5 & 6 & 21,4 \\
\hline & 12 ou mais & 6 & 7,5 & 0 & 0 \\
\hline Entrada no & Caso Novo & 52 & 65 & NA & NA \\
\hline Programa & Recidiva & 28 & 35 & 28 & 100 \\
\hline \multirow[t]{2}{*}{ AIDS } & $\operatorname{Sim}$ & 18 & 22,5 & 0 & 0 \\
\hline & Não & 62 & 77,5 & 28 & 100 \\
\hline \multirow[t]{2}{*}{ DOTs } & Sim & 20 & 25 & 2 & 7,2 \\
\hline & Não & 60 & 75 & 26 & 92,8 \\
\hline Forma de & Pulmonar & 70 & 87,5 & 26 & 92.8 \\
\hline Tuberculose & Extrapulmonar & 10 & 12,5 & 2 & 7.2 \\
\hline
\end{tabular}

Fonte: As autoras, 2017

Legenda: NA - Não se Aplica

A média de idade foi de 43,6 anos com a variação entre 18 e 77 anos. Dos 80 pacientes, $35 \%$ abandonaram o tratamento anteriormente, sendo que $10 \%$ abandonaram o tratamento mais de seis vezes. Quando analisado o nível de escolaridade dos que abandonaram o tratamento, $71,4 \%$ tinham menos de sete anos de estudo e quando analisado o nível de escolaridade dos que não abandonaram o tratamento, $40 \%$ tinham menos de sete anos de estudo. Dos pacientes que abandonaram o tratamento apenas dois possuíam a forma extrapulmonar. Da totalidade de pacientes investigados, $25 \%$ recebiam o Tratamento Diretamente Supervisionado (TDO).
Quando analisados os dados de comorbidade, a mais presente foi o HIV com 22,5\% de pacientes coinfectados. O teste de BAAR foi positivo em $82,5 \%$ dos pacientes e o teste de Cultura foi positivo em 70\% dos casos. Quanto ao Teste de Sensibilidade aos Antimicrobianos (TSA), $45 \%$ apresentaram resistência a pelo menos dois medicamentos: rifampicina e isoniazida.

\section{DISCUSSÃO}

É consenso na literatura atual associar o surgimento de linhagens de TBMR a falhas nos serviços de saúde, principalmente, no que diz respeito 
ao tratamento adequado dos casos notificados, deixando assim fluir a cadeia natural de transmissáo do bacilo $^{(7)}$. Diversas pesquisas apontam ainda a baixa adesão ao tratamento e/ou tratamento farmacológico inadequado como importantes fatores para o surgimento de $\operatorname{TBMR}^{(9)}$. No Brasil, em 2000, a taxa de abandono foi considerada alta e situou-se em torno de $17 \%{ }^{(4)}$. Neste estudo a taxa de abandono foi considerada alta quando comparada a níveis estipulados de $5 \%$ pela $\mathrm{OMS}^{(8)}$.

Dos $35 \%$ de pacientes que abandonaram o tratamento anteriormente, $10 \%$ abandonaram por mais de seis vezes. No Brasil, 96\% dos casos de resistência dos bacilos são do tipo adquirida, os pacientes infectados apresentam cavitaçóes pulmonares bilaterais em $65 \%$ dos casos, e coinfecção pelo HIV em 7\%. Para o tratamento desses casos, é necessário o uso de medicamentos injetáveis e de fármacos de reserva, com duração de 18 a 24 meses, cuja efetividade é de aproximadamente $60 \%{ }^{(13)}$. Outro fato importante é que a resistência aos medicamentos é um grande fator de risco para a falência ao tratamento instituído ${ }^{(14)}$.

Nos países em desenvolvimento, como o Brasil, as graves desigualdades sociais, o aumento da pobreza e a má distribuição de renda quando associados a precárias condiçóes médicosanitárias e, além disso, a dificuldade no acesso aos serviços de saúde -, levam às maiores taxas de infecção pelo microrganismo, ao agravamento da doença, a elevada frequência de comorbidades, coinfecção como HIV/TB, e ao desenvolvimento de linhagens resistentes a múltiplas drogas. Nesse contexto, um dos mais graves problemas da atualidade é sem dúvida a tuberculose multirresistente (TBMR) e a tuberculose extensivamente resistente (TB-XDR) ${ }^{(15)}$.

O problema da multirresistência no Brasil também é associado ao não cumprimento do esquema terapêutico. Este é um problema grave no contexto das ações de controle da $\mathrm{TB}$, uma vez que os pacientes que abandonam o tratamento da TB e/ou a terapia irregular favorecem a persistência da transmissão do microrganismo na comunidade e a resistência medicamentosa, impactando negativamente o controle da doença ${ }^{(4,7,9)}$.

A predominância de tuberculose no sexo masculino, um dos achados desta pesquisa, é semelhante ao encontrado na literatura, apontando que a diferença de acometimento de $\mathrm{TB}$ entre os sexos pode-se dever a diversos fatores,como econômicos, culturais e sociais relacionados à exposição ao bacilo ${ }^{(2,16-17)}$.

Neste estudo 25\% dos pacientes eram negros e $35 \%$ pardos, esses dados corroboram com outros estudos os quais apontam que no Brasil os negros e pardos possuem menor escolaridade, menor salário, residem nos bairros de periferia das grandes cidades e estáo excluídos de vários direitos sociais, podendo assim partir da hipótese de que o processo saúde, doença e morte é construído socialmente e demarcado pelo espaço territorial e social que homens e mulheres, brancos e negros, ocupam na sociedade ${ }^{(18-19)}$.

Sabe-se que os fatores de ordem sociocultural podem dificultar o tratamento, como o baixo nível de escolaridade, desconhecimento sobre a tuberculose e a não aceitação da doença, além do fato de alguns pacientes se considerarem curados antes da cura efetiva, já que se sentem bem ${ }^{(20)}$.

A taxa de abandono ao tratamento da tuberculose verificada nos casos analisados neste trabalho foi de 35\%. Estudos realizados no Brasil mostram índices de abandono que variam de 38\% a $42 \%$, sendo estas taxas extremamente elevadas, uma vez que o Ministério da Saúde preconiza como aceitável apenas 5\% de abandono ${ }^{(7)}$. Considerase abandono, o doente que depois de iniciado o tratamento deixou de comparecer à Unidade de Saúde por mais de trinta dias consecutivos, após data aprazada para o retorno ${ }^{(21)}$. Elevadas taxas de abandonode tratamento tambémforam encontradas em outros estudos realizados no país, mostrando a necessidade de implementação de ações que visem à sua prevenção, principalmente nos grupos que apresentam maior risco de abandono ${ }^{(2,16)}$.

O abandono do tratamento da tuberculose contribui para a manutenção da transmissão, pois doentes que náo aderem a ele ou usam 
quimioterápicos por tempo insuficiente e/ou de forma incorreta permanecem como fonte de contaminação, aumentando as taxas de recidiva e a resistência medicamentosa. Esses fatores impedem lograr a cura, uma vez que ampliam tempo e custo de tratamento ${ }^{(18,22)}$.

Nos países em desenvolvimento, $80 \%$ dos infectados encontram-se na faixa de 15 a 59 anos de idade, considerada a de maior produtividade social, com implicaçōes econômicas e sociais para o próprio indivíduo e sua famíliaa ${ }^{(23-24)}$. Neste estudo, a faixa etária mais acometida foi de 21 a 40 anos (52,5\%), a maior taxa de abandono nessa faixa pode estar relacionado ao estilo de vida desta populaçáo, que normalmente faz uso de bebidas alcoólicas e possui horários irregulares para a alimentação, fatores que podem contribuir para a interrupção do tratamento, sendo estes resultados confirmados em diversos trabalhos ${ }^{(20,25-27)}$.

Vinte por cento dos casos de doença foram encontrados na faixa acima de 61 anos, confirmando que o aumento na expectativa de vida da população tem alterado o perfil de faixa etária dos casos de tuberculose, aumentando sua ocorrência entre os idosos ${ }^{(2,16,28)}$. A localizaçáo pulmonar é a forma mais comum da TBMR corroborada no presente estudo, no qual $87,5 \%$ dos pacientes que apresentaram a forma da doença tinham a forma pulmonar ${ }^{(29,30-31)}$.

O Ministério da Saúde estima que na população geral $80 \%$ dos casos de TB apresentem a forma pulmonar ${ }^{(32)}$. Quando analisado o nível de escolaridade dos que abandonaram o tratamento, $71,4 \%$ tinham menos de sete anos de estudo e, quando analisado o nível de escolaridade dos que não abandonaram o tratamento, $40 \%$ tinham menos de sete anos de estudo, confirmando dados da literatura na qual San Pedro (2013), em um estudo de nível ecológico, discute que é possível verificar que indicadores referentes a renda, escolaridade e densidade populacional associam-se a tuberculose nos distintos níveis de agregaçáo espacial.

Diversos fatores podem dificultar $o$ tratamento e a cura da tuberculose, como por exemplo: fatores de ordem sociocultural, baixo nível de escolaridade, desconhecimento e não aceitação sobre a doença e percepção errada de considerar-se curado antes da cura efetiva, uma vez que os pacientes sentem-se melhores após os primeiros quinze dias de tratamento. Quanto menor o tempo de escolaridade, maior será a necessidade de atenção especial dos profissionais da saúde ao paciente em tratamento na promoção de orientaçóes de fácil entendimento para o paciente, evitando assim o abandono.

Segundo o Programa Nacional de Controle da Tuberculose - PNCT, estima-se, anualmente, para o Brasil, uma prevalência de 50 milhóes de infectados, 100 mil casos novos e 4 mil óbitos, e um coeficiente de incidência de 37,2/100.000 habitantes ${ }^{(33)}$. A Organização Mundial da Saúde considera, atualmente, que a TB ocupa importante percentual da utilização de recursos no setor da Saúde ${ }^{(8)}$.

Dados da Secretaria Estadual de Saúde do Rio de Janeiro informam ter ocorrido, no ano de 2014, 155 casos de multirresistência na capital fluminense ${ }^{(34)}$.

Em 2013 o Brasil registrou 503 casos de TBMR, enquanto países como Bolivia registrou 72 casos, Argentina 116 casos da doença, Paraguai apenas 6 casos de TBMR e 10 casos na Venezuela $^{(4)}$. Em 2014 o Chile registrou 15 casos de TBMR em seu país, sendo 9 dos casos de TBMR com nacionalidade chilena, 5 casos peruanos e 1 colombiano ${ }^{(35)}$.

Quando analisados os dados de casos de TBMR no Brasil em relação a países do Mercosul podemos observar uma maior prevalência de casos de TBMR no Brasil. Este cenário pode ser justificado devido a problemática do acesso ao tratamento, que é dificultado pelos atuais regimes os quais são de longa duração, mal tolerados e de difícil administração nos locais onde a maioria dos pacientes reside, motivos esses que dificultam a adesão e o sucesso do tratamento, sendo estes um dos fatores cruciais para o abandono(36). Uma das medidas é facilitar o acesso ao tratamento e, principalmente, ao Tratamento Diretamente Observado, que assim poderiamos proporcionar 
que milhares de vidas nas próximas décadas fossem preservadas. A descentralização do diagnóstico e tratamento a centros de saúde na periferia, também facilitaria a assistência aos pacientes e poderia contribuir para uma melhora nos índices de cura e para a redução da cadeia da transmissão do bacilo.

Para a redução das taxas de abandono de tratamento, é prioritário o conhecimento dos fatores associados a esse abandono, no sentido de reorientar as pessoas em tratamento de $\mathrm{TB}$ para práticas e cuidados de saúde ${ }^{(28)}$. Na cidade do Rio de Janeiro, as formas de multirresistência vêm se instalando e SE disseminando entre os diversos grupos populacionais, favorecida pelo crescimento dos adensamentos comunitários, apresenta-se relacionada a "bolsóes de pobreza" locais com grande concentração de unidades habitacionais, sem acesso à ventilação e à luminosidade, como ocorre nas comunidades quadro que facilita a propagação de doenças respiratórias ${ }^{(13)}$.

$\mathrm{O}$ aumento na taxa de abandono do tratamento, a deterioração dos serviços de saúde, devido à diminuição de investimentos no setor, são problemas também enfrentados na cidade do Rio de Janeiro ${ }^{(37-38)}$.

No Brasil a escassez de trabalhos recentes sobre a epidemiologia da TBMR no país dificulta o processo de territorialização da doença com base em seus fatores determinantes e condicionantes, sejam eles sociais, culturais ou clínico-epidemiológicos.

\section{CONSIDERAÇÕES FINAIS}

É notório que, em pleno século XXI, muitos pacientes sofrem e morrem em decorrência da TB, apesar da doença ser curável e do tratamento ser totalmente custeado pelo Sistema Único de Saúde. Envolvendo dimensões culturais, econômicas, sociais e ideológicas, este cenário tem relação com vários fatores que não se restringem apenas às políticas de saúde, motivo que dificulta o controle da doença.

Para efetivar com êxito o tratamento da TB é necessário uma organização dos serviços de saúde prestados à população, a existência de um sistema de referência e contrarreferência e o treinamento constante dos profissionais de saúde com o objetivo de uma oferta de atendimento de qualidade. Torna-se crucial a criação de vínculo com o profissional de saúde para exteriorizar os fatores que são dificultosos à adesão ao tratamento. $\mathrm{O}$ profissional de saúde, frente ao desafio de atuar com o paciente para evitar o abandono de tratamento e o desenvolvimento de formas de multirresistência, deve prestar um atendimento integral, social e cultural, sendo importante conhecer a realidade dessa população para que as intervençóes sejam acertadas e pertinentes.

$\mathrm{O}$ estudo verificou que a identificação precoce de pacientes que apresentam maior risco para abandono do tratamento justifica uma abordagem cuidadosa a ser definida conforme as condições próprias de cada local. A adesão ao tratamento, o diagnóstico correto e a terapia adequada não são importantes somente para o estado de saúde dos doentes, mas constituem também um problema de saúde pública.

O estudo confirma a necessidade de criação de um modelo de assistência voltado ao perfil dos usuários que abandonam o tratamento que, com uma prática participativa e coletiva, com grupos educativos, promova açóes preventivas, identificando e estudando estratégias para a superação de barreiras ligadas à realidade de vida, individual e coletiva. Sendo assim, espera-se que os resultados deste estudo possam contribuir para a melhoria das ações de saúde, fornecendo subsídios para as equipes intensificarem ações educativas e de conscientização quanto à necessidade de seguir o tratamento até a cura, diminuindo os casos de abandono do tratamento e, consequentemente, reduzindo os casos de TBMR.

\section{REFERÊNCIAS}

1. Ribeiro SA, Amado VM, Camalier AA, Fernandes MAA, Schenkman S. Estudo casocontrole de indicadores de abandono em doentes com tuberculose. J Pneum [Internet]. 2000 [citado 2018 Nov 05]; 26(6):291-6. Disponível 
em: http://www.jornaldepneumologia.com.br/ PDF/2000_26_6_2_portugues.pdf.

2. Paixão LMM, Gontijo ED. Perfil de casos de tuberculose notificados e fatores associados ao abandono, Belo Horizonte, MG. Rev Saúde Pública [Internet]. 2007 Apr [citado 2018 Nov 05] ; 41(2): 205-213. Disponível em: $\quad$ http://dx.doi.org/10.1590/S003489102007000200006.

3. Mendes AM, Fensterseifer LM. Tuberculose: porque os pacientes abandonam o tratamento?. Bol Pneumol Sanit [Internet]. 2004 Abr [citado 2018 Nov 05] ; 12(1): 27-38. Disponível em: http://scielo.iec.gov.br/ scielo.php?script $=$ sci_arttext $\&$ pid $=$ S0103460X2004000100005\&lng=pt.

4. Brasil. Ministério da Saúde. Secretaria de Vigilância em Saúde. Detectar, tratar e curar: desafios e estratégias brasileiras frente à tuberculose [Internet]. Brasília (DF): Ministério da Saúde 2015 [citado 2018 Nov 05]; 46(9): 1-19. Disponível em: http://portalarquivos. saude.gov.br/images/pdf $/ 2015 / \mathrm{marco} / 25 /$ Boletim-tuberculose-2015.pdf

5. Brasil. Ministério da Saúde. Secretaria de Vigilância em Saúde. Especial tuberculose [Internet]. Brasília(DF): Ministério da Saúde. 2012 Mar [citado 2018 Nov 05]. Disponível em: http://portalarquivos2.saude.gov.br/ images/pdf/2014/julho/23/BE-2012-43-Mar-o---Especial-Tuberculose.pdf.

6. Couto DS, Carvalho RN, Azevedo EB, Moraes MN, Pinheiro PGOD, Faustino EB. Fatores determinantes para o abandono do tratamento da tuberculose: representaçóes dos usuários de um hospital público. Saúde debate [Internet]. 2014 Sep [citado 2018 Nov 05] ; 38(102): 572-581. Disponível em: http://www.scielo. $\mathrm{br} /$ scielo.php?script $=$ sci_arttext $\&$ pid=S0103$11042014000300572 \& \operatorname{lng}=$ en .

7. World Health Organization. Global tuberculosis report 2015. Geneva: WHO; 2015 [cited 2018 Nov 05]. Available from: http://apps.who.int/iris/bitstream/ handle/10665/191102/9789241565059_eng. pdf? sequence $=1$ \&isAllowed $=\mathrm{y}$.

8. World Health Organization. Global Tuberculosis Report 2016. Geneva: WHO; 2016 [cited 2018 Nov 05]. Available from: http://apps.who.int/medicinedocs/ documents/s23098en/s23098en.pdf.

9. Gupta M, Saibannavar AA, Kumar V. Household symptomatic contact screening of newly diagnosed sputum smears positive tuberculosis patients - An effective case detection tool. Lung India [Internet] 2016 [cited 2018 Nov 05];33:159-62. Available from: http://www.lungindia.com/text. asp?2016/33/2/159/177445

10. Matteelli A, Roggi A, Carvalho ACC. Extensively drug-resistant tuberculosis: epidemiology and management. Clinical Epidemiology [Internet]. 2014 [cited 2018 Nov 05]; (6): 111-118. Available from: https://www.ncbi.nlm.nih.gov/ pubmed/24729727.

11. Amaral L, Vansoolingen D. A Novel Advanced Laboratory Diagnosis to Guide Tuberculosis Drug Therapy. Recent Pat Antiinfect Drug Discov [Internet]. 2015 [cited 2018 Nov 05]; 10(2):71-3. Available from: https://www.ncbi.nlm.nih.gov/ pubmed/26235217.

12. World Health Organization. Global Tuberculosis Report 2014. Geneva: WHO; 2014 [cited 2018 Nov 05]. Available from: http://apps.who.int/iris/bitstream/ handle/10665/137094/9789241564809_eng. pdf?sequence $=1$ \&isAllowed $=y$.

13. Brasil. Ministério da Saúde. Relatório Anual Programa Nacional Controle da Tuberculose Relatório de Avaliação Externa da OMS ao PNCT [Internet]; 2014 [citado 2018 Nov 05]. Disponível em: www.misau.gov.mz/.../ tuberculose/situacao_da_tuberculos.2014.

14. Ferreira KR, Cavalcante EGR, Guanilo M.C, Berti RAL, Bertolozzi MR. Portadores de tuberculose multirresistente em um centro de referência: perfil sócio-demográfico e clínico- 
epidemiológico. Rev esc enferm USP [Internet]. 2011 Dec [citado 2018 Nov 05] ; 45(spe2): 1685-1689. DOI: http://dx.doi.org/10.1590/ S0080-62342011000800008.

15. Barbosa DRM, Almeida MG, Martins LM. Aspectos socioculturais da tuberculose e diálogo com políticas públicas em saúde no Brasil [Internet]. Revista Eletrônica Gestão \& Saúde: Edição Especial; 2013 [citado 2018 Nov 05]; 2135-45. DOI: http://dx.doi.org/10.18673/ gs.v1i1.22983.

16. Andrade HS, Amaral JL, Fonseca DF, Oliveira VC, Gontijo TL, Guimaraes EAA. Clinical epidemiological features of new tuberculosis cases. Journal of Nursing UFPE [Internet]. 2016 [cited 2018 Nov 05]; 10(7). Available from: https://periodicos.ufpe.br/revistas/ revistaenfermagem/article/view/11311/12987.

17. Silva CCAV, Andrade MS, Cardoso MD. Fatores associados ao abandono do tratamento de tuberculose em indivíduos acompanhados em unidades de saúde de referência na cidade do Recife, Estado de Pernambuco, Brasil, entre 2005 e 2010. Epidemiol Serv Saúde [Internet]. 2013 Mar [citado 2018 Nov 05] ; 22(1): 77-85. Disponível em: http://scielo.iec.gov. br/scielo.php?script=sci_arttext\&pid=S167949742013000100008\&lng=pt.

18. Basta PC, Marques M, Oliveira RL, Cunha EAT, Resendes APC, Santos RS. Rev Saúde Pública. 2013;47(5):854-64

19. San Pedro A, Oliveira RM. Tuberculose e indicadores socioeconômicos: revisão sistemática da literatura. Rev Panam Salud Publica [Internet]. 2013 [citado 2018 Nov 05]; 33(4):294-301. Disponível em: http:// www.scielosp.org/scielo.php?script $=$ sci arttext\&pid=S1020-49892013000400009.

20. Giroti SKO, Belei RA, Moreno FN, Silva FS. Perfil dos pacientes com tuberculose e os fatores associados ao abandono do tratamento. Cogitare Enferm [Internet]. 2010 [citado 2018 Nov 05]; 15:271-7. Disponível em: https://revistas.ufpr. $\mathrm{br} /$ cogitare/article/view/17860.
21. Micheletti VCD, Moreira JSM, Ribeiro MO, Kritski AL, Braga JU. Drug-resistant tuberculosis in subjects included in the Second National Survey on Antituberculosis Drug Resistance in Porto Alegre, Brazil, - J bras pneumol [Internet]. 2014 Apr [cited 2018 Nov 05] ; 40(2): 155-163. Available from: http://www.scielo.br/ scielo.php?script=sci_arttext $\&$ pid $=$ S180637132014000200155\&lng=en.

22. Orofino RL, Brasil PEA, Trajman A, Schmaltz, CAS, Dalcolmo M, Rolla VC. Preditores dos desfechos do tratamento da tuberculose. $\mathrm{J}$ bras pneumol [Internet]. 2012 Feb [citado 2018 Nov 05] ; 38(1):88-97. Disponível em: http://www.scielo.br/ scielo.php?script $=$ sci_arttext $\&$ pid $=$ S180637132012000100013\&lng=en.

23. Ferreira SMB, Silva AMC, Botelho C. Tratamento da tuberculose pulmonar em Cuiabá, Mato Grosso, Brasil (19982000): distribuição espacial. Epidemiol Serv Saúde [Internet]. 2004 [citado 2018 Nov 05], vol.13, n.3, pp.175-184. ISSN 1679-4974. DOI: $\quad$ http://dx.doi.org/10.5123/S167949742004000300004.

24. Gonçalves BD, Cavalini LT, Valente JG. Monitoramento epidemiológico da tuberculose em um hospital geral universitário. J. Bras. Pneumol [Internet]. 2010 [citado 2018 Nov 05]; 36(3):347-355. Disponível em: http://www. scielo.br/pdf/jbpneu/v36n3/v36n3a13.pdf.

25. Hijjar MA. Tuberculose. J. Pneumologia [Internet] .1999 [citado 2018 Nov 05]; 25 (5): 265-266. Disponível em: file://C:/Users/not/ Downloads/1999_25_5_8_portugues.pdf.

26. Pontali E, D'Ambrosio L, Centis R, Sotgiu G, Migliori GB. Multidrugresistant tuberculosis and beyond: an updated analysis of the current evidence on bedaquiline. Eur Respir J [Internet]. 2017 Mar [cited 2018 Nov 05]; 49-3. Available From: http://erj. ersjournals.com/content/49/3/1700146.

27. Droznin M, Johnson A, Johnson AM. Multidrug resistant tuberculosis in prisons located in 
former Soviet countries: a systematic review. Plos One [Internet]. 2017 [ cited 2018 Nov 05]; 12(3). Available From: https://journals. plos.org/plosone/article? id=10.1371/journal. pone. 0174373 .

28. Chirinos NEC, Meirelles BHS. Fatores associados ao abandono do tratamento da tuberculose: uma revisão integrativa. Texto \& Contexto Enfermagem [Internet]. 2011[citado 2018 Nov 05]; 20(3):399-406. Disponível em: http://www.redalyc.org/articulo. oa?id=71421157023.

29. Gonçalves BD, Cavalini LT, Valente JG. Monitoramento epidemiológico da tuberculose em um hospital geral universitário. J Bras Pneumol [Internet]. 2010 [citado 2018 Nov 05]; 36(3):347355. Disponível em: http://www.scielo.br/ scielo.php?script=sci_arttext \&pid=S180637132010000300013\&lng=en.

30. Hino P, Cunha TN, Villa TCS, Santos, CB. Perfil dos casos novos de tuberculose notificados em Ribeirão Preto (SP) no período de 2000 a 2006. Ciênc saúde coletiva [Internet]. 2011 [citado 2018 Nov 05] ; 16(1): 12951301. Disponível em: http://www.scielo.br/ scielo.php?script $=$ sci_arttext $\&$ pid $=$ S1413$81232011000700063 \& \operatorname{lng}=$ en .

31. Vieira AA, Ribeiro SA. Abandono do tratamento de tuberculose utilizando-se as estratégias tratamento auto-administrado ou tratamento supervisionado no Programa Municipal de Carapicuíba, São Paulo, Brasil. J bras pneumol [Internet]. 2008 Mar [citedo 2018 Nov 05] ; 34(3): 159166. Disponível em: http://www.scielo.br/ scielo.php?script $=$ sci_arttext $\&$ pid $=S 1806$ $37132008000300006 \& \operatorname{lng}=\mathrm{en}$.

32. Fundação Oswaldo Cruz; Escola Nacional de Saúde Pública Sérgio Arouca. Controle da tuberculose: uma proposta de integração ensinoserviço. Rio de Janeiro: FIOCRUZ [Internet]. 2008 [citado 2018 Nov 05]. Disponível em: http://www.saude.mt.gov.br/upload/ documento/81/controle-da-tuberculose-umaproposta-de-integracao-ensino-servico-\%5B81080909-SES-MT\%5D.pdf.

33. Brasil. Ministério da Saúde. Secretaria de Vigilância em Saúde. Perspectivas brasileiras para o fim da tuberculose como problema de saúde pública. Brasília (DF): Minitério da Saúde [Internet]. 2016 [citado 2018 Nov 05]. Disponível em: http://portalarquivos2.saude. gov.br/images/pdf/2016/marco/24/2016-009Tuberculose-001.pdf.

34. Secretaria de Vigilância à Saúde. Sistema de Informação de Agravos de Notificação - Sinan. Normas e Rotinas. Brasília: Ministério da Saúde, 2002.

35. Chile. Ministério da Salud. Tuberculosis Informe de Situación Chile 2014. Programa Nacional de Control y Eliminación de la Tuberculosis. Santiago: Ministério da Salud [Internet], 2015 Jun [cited 2018 Nov 05]. Available from: https://diprece.minsal.cl/wrdprss_minsal/wpcontent/uploads/2015/09/Informe_tbc_2014. pdf.

36. Rabahi MF, Rodrigues AB, Mello FQ, Neto JCA, Kritski AL. Noncompliance with tuberculosis treatment by patients at a tuberculosis and AIDS reference hospital in midwestern Brazil. Braz J Infect Dis [Internet]. 2002 Apr [cited 2018 Nov 05]; 6(2): 6373. Available from: http://www.scielo.br/ scielo.php?script=sci_arttext $\&$ pid $=$ S1413$86702002000200002 \& \operatorname{lng}=$ en .

37. Ferraz AF, Valente JG. Epidemiological aspects of pulmonary tuberculosis in Mato Grosso do Sul, Brazil. Rev Bras Epidemiol [Internet]. 2014 Mar [cited 2018 Nov 05]; 17 (1). Available From: http://www.scielo. $\mathrm{br} /$ scielo.php?script=sci_arttext $\&$ pid $=S 1415$ $790 X 2014000100255 \& \operatorname{lng}=\mathrm{en}$.

38. Krauss MR., Harris DR, Abreu T, Ferreira FG., Ruz NP, Worrell C et al . Tuberculosis in HIVinfected infants, children, and adolescents in Latin America. Braz J Infect Dis [Internet]. 2015 Feb [cited 2018 Nov 05] ; 19(1): 
23-29. Available from: http://www.scielo.br/ scielo.php?script $=$ sci_arttext $\&$ pid $=S 1413$ 86702015000100023\&lng=en.

Recebido em: 04/04/2018.

Aprovado em: 07/12/2018. 